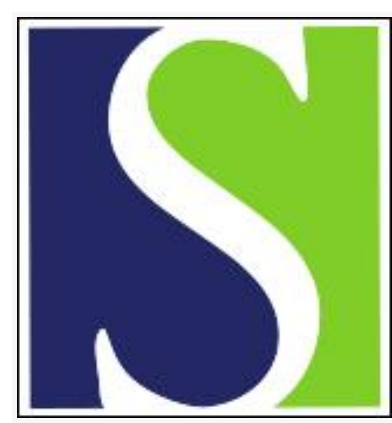

Scand J Work Environ Health 1990;16(6):445-447

https://doi.org/10.5271/sjweh.1759

Issue date: 01 Dec 1990

Re: "Triazine herbicides and ovarian epithelial neoplasms" by A Donna, P Crosignani, F Robutti, PG Betta, R Bocca, N Mariani, F Ferrario, R Fissi, F Berrino Scand J Work Environ Health 1989. by Minder CE

This article in PubMed: www.ncbi.nlm.nih.gov/pubmed/2284596

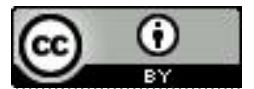




\section{Re: "Triazine herbicides and ovarian epithelial neoplasms" by A Donna, P Crosignani, F Robutti, PG Betta, R Bocca, N Mariani, F Ferrario, R Fissi, F Berrino Scand J Work Environ Health 1989; $15: 47-53$}

The paper "Triazine Herbicides and Ovarian Epithelial Neoplasms" by Donna et al (Scand $J$ Work Environ Health 1989;15:47-53) cannot, in my opinion, go uncommented upon. The topic of causation of cancer through triazines is, in view of the widespread use of this herbicide, of very great health importance and should be dealt with carefully. My comments concern the following three points: statistical aspects, the classification of the exposed women, and possible confounders and biases.

The statistical results of the paper consist of a significant odds ratio of $2.7(\mathrm{P}=0.10)$ and two positive trends with growing exposure with $\mathrm{P}$-values of $5 \%$ and $8 \%$, respectively. Scanning the paper for the number of tests performed, one finds that there were at least 47; probably there were more. Two tests concerned the well-known dependency of ovarian cancer risk on the number of births. They turned out to be fairly significant. In the remaining 45 tests one would expect (under the hypothesis of no effects and at a significance level of 0.1$) 4.5$ significant results. There were four. From this point of view, the evidence presented is rather meager. A second way of assessing the significance of the results presented is to use adjustments for the number of tests performed. On the assumption that there were 20 essentially separate tests, the Bonferroni correction amounts to a factor of 0.05 , and no significant result remains. Both analyses show that the results concerning statistical significance are not beyond doubt; in other words, they could be due to chance.
The second aspect covers the classification of the definitely exposed subjects. From the list at the end of the paper, it is possible to devise stricter definitions of "definitely exposed." I have recalculated the crude odds ratios for the women reporting to have "used triazine herself" and "used triazine herself before 1978." The latter category was used to allow for some (probably too short) latency for the ovarian cancer to develop. The resulting odds ratios are 2.3 for the definition used in the report, 1.50 for those reporting to have used triazine themselves, and 0.90 for those reporting to have used triazine themselves before 1978. This is a decreasing sequence of odds ratios, while in a situation of increasingly strictly defined exposures one would expect an increasing sequence. It is not easily possible to test for the significance of this decreasing trend, so there may, too, be no trend. Explanations for "no trend" are possible, but all coming to my mind are implausible.

Although the authors maintain that the study has been designed with the aim of minimizing selection bias, there are at least two types of bias which could have produced the observed results and which were not discussed and not controlled for adequately. In view of the severity of the disease of ovarian cancer and of the long average lag time of 30 months between diagnosis and interview, it is very possible that there was an interviewer bias due to the fact that the interviewer could visually distinguish the cases from the referents. This possibility is not raised and discussed in the paper. A second possible bias could be due to the in- 
fluence of socioeconomic factors. The Alexandria Hospital register is not a recognized area-covering cancer register. (It is to my knowledge, for example, not included in Cancer in Five Continents.) It is therefore possible that women with ovarian cancer from betteroff families were missed due to their seeking treatment in one of the bigger centers of northern Italy. This possibility would presumably lead to the effect observed in that lower socioeconomic strata with inherently higher triazine exposure would also seem to have more ovarian cancer. In addition, Berkson's bias could be operating in this case. It is well known that hospitalized patients often have higher exposure values than unhospitalized ones. As the cases in this study were at least at some time hospitalized and the referents were collected from the general population, this is a possibility.

If all of these points are taken into consideration and the most weight is placed on the statistical aspect, one cannot but conclude that the case for an association between ovarian cancer and triazines is weak indeed. The case is even weaker for a causal link.

\section{Christoph E Minder, $\mathrm{PhD}$}

Department of Social and Preventive Medicine University of Berne

Finkengubelweg 11

CH-3012 Berne Switzerland

\section{Authors' reply}

The first comment of Dr Minder's is that the significance of triazine-related results would be a simple reflection of the number of tests performed.

As we stated at the beginning of our paper, the study was conducted "to evaluate the specific role of triazines in ovarian carcinogenesis... [p 47]." The hypothesis to investigate was suggested by a former case series, in which six of eight cases with definite exposure to herbicides involved exposure to triazines, and it was corroborated by a hospital-based case-referent study. This was the sole hypothesis to test, and the only pertinent statistical tests of this question were the significance of relative risks and the significance of trend, all in favor of a positive association between triazine exposure and ovarian cancer. In particular, the onetailed $\mathrm{P}$-value (the other tail of the distribution being irrelevant to the question) for the risk of 2.7 associated with "definite exposure" was 0.05 ; not 0.1 as stated by Dr Minder.

The role of reproductive factors was analyzed and reported only to elucidate potential confounders to be taken into account in the analysis of triazine-related risks. The study was not a "fishing expedition," nor was it planned to explore the effect of various reproductive factors on ovarian carcinogenesis. The issue of multiple comparison, to which Dr Minder gives the most weight, is totally nonsense.

The second point raised by Dr Minder regards our criteria for the attribution of the exposure. He does not comment on the criteria as a whole but, instead, proposes a different classification for "definitely exposed" subjects.

Dr Minder's criteria are such that those subjects who reported personal preparation and distribution of triazines are thought to be more exposed than those who worked in fields treated with such herbicides and that the duration of exposure is irrelevant to the risk. As we stated in the paper, on page 48 , triazine herbicides are stable chemicals whose mechanisms of human absorption are unknown and, in addition, the preparation and distribution of such herbicides usually occurred once or twice a year. We had no reason to suppose that the preparation and distribution of these herbicides would lead to a cumulative exposure higher than that occurring during work carried out in the fields where the herbicides were used. Moreover, all the subjects who prepared and distributed triazine herbicides also worked in the treated fields. Therefore, it seemed reasonable to consider these instances of exposure in a single category formed of individuals for whom there was almost certain exposure, although not quantifiable, and to use the length of such exposure as an indicator of cumulative dose.

As far as the risks computed by Dr Minder are concerned, we should clarify that, in the appendix, we reported only modalities of definite exposure.

The two exposed cases whose "definite exposure" began after 1978 also had a prolonged "possible exposure." Case BB worked in corn and wheat fields from. 1959 to 1984 , and case RR worked in corn and wheat fields from 1948 to 1986. Referent MP had only the exposure that was reported in the appendix. Nonetheless, we were forced to consider these cases as "definitely exposed" for a short period by the criteria we established before the analysis (end of paye 48). Changing the criteria after knowing the disease status of the subjects would have given rise to the possibility that some results would be due to the adaptation of the analysis to the data.

The third point raised by Dr Minder is on the comparability of the information between the cases and referents due to interviewer bias. Appointments with the subjects to be interviewed were not made by the interviewer herself, and she was "unaware of the disease status prior to, and sometimes even after, the completion of the interview [p 48]."' Other elements in favor of the comparability of the information are discussed at the beginning of the section "Data Collection" on page 51 of the report.

Although one can almost always advocate interviewer bias in the interpretation of case-referent studies with anamnestic assessment of exposure, the procedure we used for organizing the interview and all the elements scrutinized are against this hypothesis. 
In addition, at the time of the data collection, no information had ever appeared in the media on the carcinogenic effects of herbicides. We intentionally omitted chemical species and trade names of herbicides in the first paper (1), which described the hospitalbased case-referent study, to allow us to explore this hypothesis further at the population level. As far as we know, the first paper has never been cited in the general press. The public attention to the problem of the pollution of drinking water by atrazine, as detected by analytical methods, was aroused when we were terminating the data collection but, even then, the potential carcinogenicity of atrazine was not mentioned. One should also remember that the interview concerned any kind of herbicidal use, without any particular emphasis on triazines.

A fourth point was raised about the completeness of the case series. As population representativity is not the objective of the Alessandria hospital-based cancer register (as it is for other similar registers), we conducted a survey to establish the institution to which ovarian cancer patients of the area are referred. This survey led us to consider (in addition to the Alessandria cancer register, which contains reports from eight hospitals in the Province) "the archives of 10 other hospitals [p 48]." The completeness of the case series was thus probably achieved, as discussed in the section "Population Representativity" on page 50. As we compared incident cases with their source population, there is no room for invoking the possibility of Berkson bias.

The topic of causation of cancer through triazine is, in view of the widespread use of these herbicides, of very great health importance, as Dr Minder pointed out. Atrazine, the triazine most used in our area, has shown mutagenic (2) and experimental carcinogenic potential (3), and other epidemiologic evidence is available for triazine exposure (4). Thus we hope that our reply will be useful to all those whose concern is public health and safety.

\section{References}

1. Donna A, Betta PG, Robutti F, Crosignani P, Berrino F, Beliingeri D. Ovarian mesothelial tumors and herbicides: a case-control study. Carcinogenesis 1984;5:941-2.

2. Adler ID. A review of the coordinated research effort on the comparison of test systems for the detection of mutagenic effects, sponsored by the E.E.C. Mutat Res 1980; 74:77-93.

3. Italian National Commission for Toxicology. Draft for hearing; evaluation of a first list of pesticides. Rome: Istituto Superiore di Sanita', 1989. (CCTN/Pesticidi/5/89.)

4. Hoar SK, Blair A, Holmes FF, et al. Agricultural herbicide use and risk of lymphoma and soft-tissue sarcoma. JAMA 1986;256:1141-7.

Paolo Crosignani, DSc, $\mathrm{MD}^{1}$, Adalberto Donna, PhD, ${ }^{2}$ Franco Berrino $\mathrm{MD}^{1}$

${ }^{1}$ Epidemiology Unit, National Cancer Institute, Milano, Italy.

2 Department of Pathology, City Hospital-USSL, Alessandria, Italy.

(Address for correspondence: Dr P Crosignani, Epidemiology Unit, National Cancer Institute, Via Venezian, I-20133 Milano, Italy) 